Presented at the International Conference on Bubble Chamber Technology, Argonne National Laboratory, June 10-12, 1970

\section{UCRL -19870 \\ Preprint \\ CONA-700623 MATÉ}

BUBBLE CHAMBER PHYSICS IN THE SEVENTIES

G. H. Trilling

July 1970

AEC Contract W $-7405-\mathrm{eng}-48$

THIS DOCUMENT CONFIRMED AS

UNCLASSIFIED

DIVISION OF CLASSIFICATION

BY

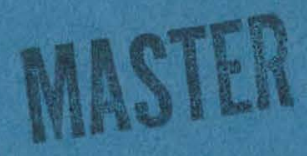

DATE $1 / 10 / 5 / 70$

\title{
LAWRENCE RADIATION LABORATORY
} UNIVERSITY of CALIFORNIA BERKELEY 


\section{DISCLAIMER}

This report was prepared as an account of work sponsored by an agency of the United States Government. Neither the United States Government nor any agency Thereof, nor any of their employees, makes any warranty, express or implied, or assumes any legal liability or responsibility for the accuracy, completeness, or usefulness of any information, apparatus, product, or process disclosed, or represents that its use would not infringe privately owned rights. Reference herein to any specific commercial product, process, or service by trade name, trademark, manufacturer, or otherwise does not necessarily constitute or imply its endorsement, recommendation, or favoring by the United States Government or any agency thereof. The views and opinions of authors expressed herein do not necessarily state or reflect those of the United States Government or any agency thereof. 


\section{DISCLAIMER}

Portions of this document may be illegible in electronic image products. Images are produced from the best available original document. 
Talk presented at the International Conference on Bubble Chamber Technology, Argonne National Laboratory, June 10-12, 1970

\title{
BUBBLE CHAMBER PHYSICS IN THE SEVENTIES
}

\author{
G. H. Trilling \\ Department of Physics and Lawrence Radiation Laboratory \\ University of California, Berkeley, California 94720
}

This meeting, devoted to a discussion of recent technical developments in the bubble chambers, has been to some extent what my old friend, Don Glaser, used to call a plumber's meeting. Actually this is not quite true since the physics applications were always close to the discussion even when devoted to purely technical matters. It is not my intention, in the present talk, to repeat or summarize everything that was said in the last few days. You have heard it once and there would be little purpose in repeating it now. Rather I would like to emphasize the relation between the technical developments and the physics results likely to come from bubble chambers in future years.

Before I start, however, I want to address two remarks to our hosts. First, I am surc that all the attendees of this Conference join me in congratulating the ANL bubble chamber group for its magnificent achievement in successfully operating the world's largest bubble chamber and superconducting magnet. Second, on behalf of all of us, I want to thank our hosts for their hospitality and all the excellent arrangements they have made to make this Conference interesting and enjoyable.

\section{RECENT BUBBLE CHAMBER WORK}

Before saying something about the future it is perhaps worth examining the recent past and see where we are now. Therefore I want to begin my 
discussion with some examples of recent interesting, bubble chamber experiments. Since there are many bubble chamber builders in the audience, it is appropriate to show how far their efforts up to now have taken us in the ability to do interesting experiments. Much of what they usually hear from users is in the nature of complaints; at this point, as a user, I want to say thanks for what the se efforts have made possible.

The overall bubble chamber program for all accelerators can be characterized by many kinds of figures: total photographs per year which measures in tens of millions, total events processed per year which measures in millions, particle beams available to bubble chambers which include protons to $\sim 30$ $\mathrm{GeV}$, pions to $\sim 25 \mathrm{GeV}$, kaons to $\sim 20 \mathrm{GeV}$, monochromatic polarized photons to $\sim 10 \mathrm{GeV}$, neutrons and $\mathrm{K}_{\mathrm{I}}^{0}$ over a variety of energies. More interesting than all these kinds of descriptive information concerming the capabilities of the bubble chamber program is some consideration of its recent physics contributions.

$\perp$ can categorize the se contributions in the three areas of weak, electromagnetic and strong interactions. Some selected examples from each follow:

\section{A. Weak Interactions}

\section{Decay Properties of Hyperons}

High statistics experiments at CERN, Brookhaven, and Berkeley ${ }^{l}$ using low energy $\mathrm{K}^{-}$beams (either stopping or at the $\Lambda(1520)$ energy) have yielded remarkable measurements of the non-leptonic decay parameters for hyperon decay, particularly $\Sigma^{ \pm}$decay. For example, the asymetry parameters for $\Sigma^{+} \rightarrow \mathrm{n}^{+}$and $\Sigma^{-} \rightarrow \mathrm{n}^{-}$decay quoted in the Particle Data Tables, namely $\alpha=0.068 \pm 0.016$ and $-0.078 \pm 0.020$ respectively, have statistics of truly heroic proportions in back of them. 


\section{Neutrino Interactions}

Work with the propane-filled CERN heavy liquid bubble chamber coupled to careful beam normalization has yielded some of the first quantitative data on neutrino nucleon interactions including the total cross-section measurement of $\left(0.8 \times 10^{-38} \mathrm{~cm}^{2}\right) \times E_{v} \cdot{ }^{2}$ Figure 1 shows some properties of the reaction $v p \rightarrow \mu^{-} \mathrm{p}^{+}$based on about 50 interactions on free protons. This ability to study processes involving free nucleons is the threshold to a future program which will be a major part of the activity at new high-energy accelerators.

\section{B. Electromagnetic Interactions}

Perhaps the most striking results have been the beautiful $\rho$ photoproduction experiments with the SLAC 82-inch chamber exposed to the backscattered ruby laser beam facility. The se experiments with photons of energy 2.8 and 4.7 GeV have given striking evidence for s-channel helicity conservation in the diffraction production of $\rho$ mesons. ${ }^{3}$ This is illustrated in Fig. 2 which shows $\rho$ decay angular distributions in the helicity frame and confirms the $\sin ^{2} \theta \cos ^{2} \psi$ prediction for s-channel helicity conservation. The graphs in Fig. 2 are based on about 3000 events at each momentum; these statistics, though not huge, required a very substantial exposure ( 750,000 photographs).

\section{Strong Interactions}

Strong interactions have of course been the natural area for bubble chamber research. I shall mention a very few items from recent work in this fiela:

\section{Baryon Spectroscopy}

The experimental data for the study of strange baryon resonances has almost totally come from bubble chamber formation and production experiments. 
In this connection I want to mention particularly the beautiful CERN-HeidelbergSaclay systematic study of $\mathrm{K}^{-} \mathrm{p}$ interactions below and above $I \mathrm{GeV} / \mathrm{c}$, of which a typical set of results is shown in Fig. 3 taken from a recent paper. ${ }^{4}$ when one realizes that for each channel whose cross sections are displayed in Fig. 3 there are detailed angular distributions, polarizations, etc., the total information acquired from such experiments is truly impressive.

\section{Boson Spectroscopy}

I want to first show three slides from an experiment by the A Grnup at Berkeley to indicate what is probahly near thelimit of what onc can do witl a conventional experiment. ${ }^{5}$ The exposure was a $\pi^{+} p$ run a.t. $7 \mathrm{reV} / \mathrm{c}$ at SLAC in the 82-inch chamber. Figure 4 a shows a $\pi^{+} \pi^{+} \pi^{-}$mass spectrum from 49,474 events of the type $\pi^{+} \mathrm{p} \rightarrow \pi^{+} \mathrm{p}^{+} \pi^{-}$. Figure $4 \mathrm{~b}$ shows the same for a selected subsample of about 2500 events with momentum transfer and $\Delta(1236)$ removal cuts showing a clear $A_{2}$ signal without the fine structure in the mass spectrum observed in the CERN missing mass spectrometer. 6 Figure $4 \mathrm{c}$ shows 474 events of tlie lype $\pi^{+} \mathrm{p} \rightarrow \mathrm{p}^{+} \eta$ and $\mathrm{pK}^{+} \mathrm{K}^{\mathrm{n}}$ again with a good $\mathrm{A}_{2}$ signal and no fine structure. The statistics are massive, although after cuts and in the study of the rarer channels one quickly gets down to numbers of events which are not very large.

\section{Production Mechanisms and Many-Particle Final States}

Studies of inelastic processes leading to quasi-two-body or multi-body final states have permitted tests of various models for high energy interactions. An enormous amnunt of information giving cross sections, pruduction and decay angular distributions, multi-particle correlations, etc., for a large range of energies, incident particles, and both neutron and proton targets has been accumulated by laboratories and universities all over the 
world. The development of theories and models to interpret this immense volume of information is a continuing process providing livelihood for many theorists and phenomenologists. Indeed it is the generation by bubble chamber experiments of large volumes of data on multi-body final states which has stimulated theoretical consideration of ways to analyze and understand this large amount of new information.

It is evident, but must again be emphasized that this brief consideration of present bubble chamber work is not a summary but only a mention of a few examples to give the flavor of the physics coming out. Having made this short review, one can now consider future possibilities. We can ask what sorts of development will lead to new information beyond what we now have. There are several types of answers:

(i) Increases in statistics--one of the lessons of experiment in recent years is that large statistical increases do a great deal more than reducing errors by a square root factor; they provide a degree of detail not previously available and often lead to qualitatively new phenomena. Figure 5 from the work of Coyne et al. ${ }^{7}$ gives a striking example of this; namely, a dip in the $\pi^{+} \pi^{-}$mass spectrum from the reaction $\pi^{+} \mathrm{p} \rightarrow \mathrm{p} \pi^{+} \pi^{+} \pi^{-}$at the $\omega$ mass resulting from interference between the $G$ parity violating $2 \pi$ decay mode of the $\omega$ and the $p \rightarrow \pi^{+} \pi^{-}$decay. In an earlier experiment at the same energy this effect was not observed because statistics were poorer by about an order of magnitude.

(ii) Increases in types of events accessible to study--conventional bubble chamber work with momentum analyzed incident beams permits only the study of events with at most one undetected neutral. If the incident particle is of unknown momentum, even events with one neutral are difficult to handle. The introduction, either internal or external to the bubble chamber, of devices 
to detect neutrals would greatly widen the window on particle physics provided by the bubble chamber.

(iii) Increases in energy--it is a fond hope of particle physicists that higher energies will provide new insights. These insights can come from (a) new processes with energy thresholds above presently studied energies, (b) simplification of processes already seen at present energies because of cleaner separation between resonances and background, (c) information on energy dependence over a wide range of energies of various parameters describing the processes such as cross sections, diffraction slopes, decay density matrix elements, etc.

(iv) Improved capability for neutrino physics--a major justification of the NAL accelerator is the potential for detailed studies of neutrino interactions. At present most of our knowledge of weak processes comes from studies Uf Lie decays OI à very t'inite number of particles in which momentum transfers and energies are outside our control. It is evident that a vast potential for increasing that knowledge is providen hy noutrino interaction proccuica. We now go into some detail in the discussion of techniques which may bring about the improved capabilities discussed above.

\section{TI. IYYRID SYSTEMS}

The substantial cost of film and data reduction seems to suggest that large statistical increases using conventional modes of bubblc chamber operation will not be possible in the future. For some of the simpler interactions, the procedure for going to high statistics will involve the abandonment of the bubble chamber in favor of large wire chamber spectrometers or streamer chambers. However, for many processes involving, for example, (i) many final state particles, (ii) slow recoil particles, (iii) multiple vertices, etc., 
the large solid angle and vertex visibility of the bubble chamber will still be needed. The natural direction for such work with high statistics will be the development of hybrid systems, with counters and spark chambers used both for additional information useful to the analysis and to provide a trigger for picture taking on interesting events. Thus high statistics on special event types will be available without the large volume of film usage involved if every expansion is photographed. Since the permissible number of beam tracks in a chamber in a single expansion is limited, from the point of view of accumulating sizable statistics in a finite time it is desirable to have a rapid pulsing capability. This capability is rapidly improving as a result of recent technical developments.

These developments can be summarized as follows:

(A) The ANI 30-inch chamber has been operated in a quintuple-pulsing-peraccelerator-burst mode routinely: The SLAC 40-inch chamber has been pulsed at the rate of 12 times per second for one second. As will be discussed further both of these chambers are or will soon be used in a hybrid mode.

(B) Barney et al. 8 at SLAC have operated 2-inch and 4-inch diameter hydrogen chambers at rates of 60 and $90 \mathrm{~Hz}$ with good track quality. A larger chamber, of diameter 15 inches and depth 5.5 inches, to mun at $60 \mathrm{~Hz}$ is under construction and scheduled for completion in about one year. This chamber is to be operated in conjunction with a wire chamber spectrometer to do experiments in boson spectroscopy.

Walker and collaborators at Wisconsin are also developing a chamber, of diameter 14 inches and depth 8 inches, to run at $30 \mathrm{~Hz}$.

(C) There are also longer range developments. The SLAC group is working with sonic chambers 8 : the chambers are essentially one wave length deep with 
the sensitive regions in the neighborhood of the anti-nodes. The bubbles are formed and recompressed in one single cycle. This group has operated a test hydrogen chamber at $9 \mathrm{kHz}$, wave length about $10 \mathrm{~cm}$, with a sensitive region of about $2 \mathrm{~cm}$. A track candidate has been seen. Eventually the hope is to go to frequencies below $1 \mathrm{kHz}$ with a sensitive region at least $20 \mathrm{~cm}$ deep.

The CERN group ${ }^{10}$ is working with ultrasonic chambers, at frequencies of $100 \mathrm{kHz}$ or more. Wave lengths are of the order of $\mathrm{mm}$; a chamber would contain many wave lengths giving tracks with gaps of the order of the wave length. In this mode, many cycles are required for bubble growth, and recompression is accomplished by turning off the ultrasonic excitation for perhaps $10 \mathrm{msec}$. Sensitivity in helium where only a small expansion ratio is needed has been achieved some time ago. Recently sensitivity was achieved in hydrogen, with a conventional expansion to obtain part of the pressure drop and ultrasonic. excitation at $360 \mathrm{kHz}$ to go the rest of the way.

After these delalls un rap1d pulsing chamber development, it is perhaps useful to indicate current work on the application of hybrid systems to particle physics experimenta:

(1) Two experiments have been done in the PPA rapid pulsing chamber by Cline and coworkers. Both of these experiments involved the study of bosons produced at high momentum transfers by pion-rucleon collisions, by means of a trigger based on the detection of a relatively fast forward proton. Preliminary results have been reported at the recent Washington APS Meeting. ${ }^{11}$

(2) Gunderson et al. ${ }^{12}$ have combined with the 30-inch ANL chamber a counter-spark-chamber setup designed to detect fast forward outgoing neutral particles (neutron or $K_{L}^{\circ}$ ) produced in $\pi$-nucleon interactions at several $\mathrm{GeV}$. 
This setup gives a trigger on a fast neutral unaccompanied by forward charged secondaries in about $1 / 30$ of the expansions. Besides giving this trigger, the spark chambers provide accurate directional information for the neutral secondary. A diagram of the setup is shown in Fig. 6. It is anticipated by Gunderson et al. that this arrangement will be highly effective in the study of backward boson production by $4-6 \mathrm{GeV}$ pions interacting with protons and neutrons.

(3) Another example of a hybrid system is the setup in Fig. 7 proposed for a SIAC experjment by the Cal Tech group. ${ }^{13}$ The intention is to study nucleon diffractive dissociation by detecting events produced by high energy pions or kaons in which a fast secondary, either charged or neutral, is emitted near the forward direction. To achieve this goal, the 40-inch SLAC bubble chamber is to be followed by a wire chamber spectrometer of about $1 \%$ resolution, Čerenkov counters, and gamma ray detectors. The spectrometer measures the momentum of forward, fast charged secondaries, the Čerenkov counters discriminate between pions, kaons and nucleons, and the gamma ray detectors determine the directions of photons from the decay of forward emitted $\eta$ or $\pi^{\circ}$ mesons. This electronic instrumentation is used to trigger the bubble chamber light flash on interesting events defined as nonelastic interactions producing a fast forward secondary, charged or neutral. Furthermore, for such events the bubble chamber photograph is supplemented by the precise information obtained in the spark chamber setup to permit better fitting and identification of events. It is estimated by the Cal Tech group that this trigger arrangement reduces the required picture taking by a factor of about 20. Furthermore, there is, of course an enormous saving in data analysis effort. 
(4) Other hybrid arrangements either being installed or under active consideration involve placement of scintillation or solid state counters either inside or in very close proximity to bubble chambers for a variety of purposes such as: (i) time-of-flight measurements for incident $\mathrm{K}_{\mathrm{L}}^{\mathrm{O}}$ mesons, (ii) determination that a beam track has interacted, (iii) detection of multiparticle final states. Presumably more details on these are given elsewhere in the Conference Proceedings, and they will not be further discussed here. ${ }^{14}$

To conclude this discussion of hybrid systems, a few general comments, not all orlginal with me, are in order:

(a) As stressed by Walker, hybrid systems have thus far been only marginally useful. In part, this is because of the difficulty of obtaining really useful triggers, given the geometrical boundary conditions imposed by a complicated device like a bubble chamber.

(u) Beslues ylving more events per picture taken or sometimes instead of that, counters and spark chambers can provide additinnal. data useful in making fits. There is almost no experience on this as yot.

(c) At high energies, with more collimation of secondaries, hybrid systems will probably be easier to design.

\section{TRACK SENSITIVE TARGETS}

The inability of bubble chambers to handle multi-neutral events has seriously limited the study of certajn classes of inelastic processes. As the incident energy increases, the fraction of such events also increases markedly with the following deleterious consequences: (i) only a small parl of the total cross section is accessible to complete event reconstruction providing a rather incomplete picture of inelastic processes; (ii) since the fittable events are few, the measuring efficiency in terms of the ratio of 
such events to total events measured is low. Finally, at high energies even the events with only one missing neutral are likely to be subject to serious ambiguity unless that neutral can be detected.

In the past, bubble chamber experiments requiring the detection of $\pi^{\circ}$ have used propane-freon mixtures and more recently hydrogen-neon mixtures. For example, a Berkeley-Brookhaven-Orsay-Milan-Saclay collaborative experiment to study coherent $\mathrm{K}^{-}$interactions in neon has been able to process such events as

$$
\mathrm{K}^{-}+\mathrm{Ne} \rightarrow \mathrm{Ne}+\mathrm{K}^{-}+\pi^{\circ}+\pi^{0}
$$

This experiment is done in the BNL 80-inch chamber filled with a mixture of 2/3 neon, $1 / 3$ hydrogen (atomic proportions), radiation length $43 \mathrm{~cm}$. Figure 8 shows the $\pi^{0} \rightarrow r+\gamma$ mass spectrum reconstructed from energy and angular measurements on the converted photons. The $\pi^{\circ}$ peak has a full width of about $30 \mathrm{MeV}$, from which the energy uncertainty for each gama ray is about $\pm 15 \%$. For different mixtures, the energy error would be expected to scale roughly in inverse proportion to the radiation length.

For the future, the development which gives the best promise of providing good detcction of multi- $\pi^{\circ}$ events produced on free nucleons is the track sensitive target first developed by DESY and CERN. ${ }^{15}$. In this device, one fills the target with hydrogen or deuterium as required by the physics, and uses a neon-hydrogen mixture in the outer volume to provide photon conversion. Recent development work on this technique has been carried on at CERN. in collaboration with the Rutherford Laboratory, at SLAC and at BNL.

The CERN group has mounted a plexiglas target of rectangular cross section inside the British 1.5-m hydrogen chamber at the Rutherford Laboratory. The target is $1.4 \mathrm{~m}$ long, $30 \mathrm{~cm}$ high and $4 \mathrm{~cm}$ deep, with a wall thickness of $2 \mathrm{~mm}$. 
There is no attempt to maintain any pressure or temperature differential between the target and the external volume. During expansion the large walls flex about $150 \mu$. For an actual experiment to study $\Lambda^{\circ}$ beta decay, the volume outside the target was filled with a neon-hydrogen mixture of radiation length $70 \mathrm{~cm}$. Satisfactory sensitivity has been obtained simultaneously in the target and in the surrounding volume. Because of a variety of technical difficulties actual data taking on a production basis has yet to come; there is very little information on distortions and, generally, on practical problems of reconstruction and experimental analysis. The CERN group is also contemplating the construction of a similar target for the Big European Bubble Chamber $(B E B C)$. Such a target which might be 2 to $2.5 \mathrm{~m}$ long, $1 \mathrm{~m}$ wide and $10 \mathrm{~cm}$ deep is illustrated in Fig. 9.

Both the SLAC and BNL groups have taken a somewhat different approach lu lrack-sensitive-target design. Because of differences between neon and hydrogen vapor pressure curves the single arrangemont of equal temperulure and pressure inside and outside the target somowhat limits the l'lexib1lity in the choice of mixture proportions to achieve simultaneous sensitivity. A hydrogen target cannot mun with pure neon in the external volume for cxample. Consequently, to provide more flexibility and more adjustment, both SLAC and BNL have designed targets which could maintain both pressure differentials and temperature differentials between the two sensitive volumes. In both cases plexiglas cylinders are used with somewhat elaborate bellows arrangements permitting a higher pressure in the target and cooling loops to allow a lower temperature in the target. Figure 10 shows the target arrangement being constructed for the BNL 80-inch chamber, namely a plexiglas cylinder $20 \mathrm{~cm}$ in diameter and $1.45 \mathrm{~m}$ long. If this arrangcment is successful, a number of experiments will probably be run with it early next year. For the 
NAL chamber, discussed in the next section, a target consisting of a cylinder $3 \mathrm{~m}$ long and $i .6 \mathrm{~m}$ in diameter is contemplated. It is useful to note here that it is not strictly necessary to convert all gammas for a successful fit. In a two- $\pi^{\circ}$ event, energy and angle measurements for all gammas give a $6 \mathrm{C}$ fit, and in the absence of one converted gamma a $3 \mathrm{C}$ fit is still possible.

In conclusion, it is perhaps appropriate to stress the following points:

(a) No one has yet successfully done a real physics experiment with a track-sensitive-target even though they have existed in principle for several years. The problems of distortion, reconstruction, etc. have not had the test of a real physics problem. It is therefore fair to say that considerable development still lies ahead.

(b) For the reasons stated at the beginning of this section the real value of the bubble chamber technique for hadron physics at very high energy may crucially depend on the success of the track-sensitive-target technique.

\section{I.V. LARGE BUBBLE CHAMBERS}

A number of large bubble chambers are being constructed, tested or, as in the case of the ANL 12-foot chamber, are ready to do some physics. Table I contains some information on the se chambers.

I believe it is fair to say that the real justification for building chambers in the $20-100 \mathrm{~m}^{3}$ volume range is the neutrino physics. While it is extremely likely that with such chambers, improved hadron physics experiments can be carried out, it is not entirely clear that if hadron physics were the only justification the investment for a very large chamber would be an optinal use of limited funds. Indeed I think that whether such an investment were optimal or not hinges substantially on the successful usage of track-sensitive targets. I shall now consider separately the applications to neutrino and hadpur phyșics. 
Table I. Large Chambers

\begin{tabular}{|c|c|c|c|c|}
\hline Name & Type & Dimensions & & $\begin{array}{c}\text { Approximate } \\
\text { date of operation }\end{array}$ \\
\hline ANL 12-foot & Hydrogen & $\begin{array}{l}\text { 12-foot diameter } \\
7 \text {-foot height }\end{array}$ & $\therefore$ & Now \\
\hline Gargamelle & Heavy liquid & $\begin{array}{l}\text { 4.8-m length } \\
1.9-\mathrm{m} \text { diameter }\end{array}$ & $\because \quad .$. & $\begin{array}{l}\text { Experiment in } \\
\text { spring } 1971\end{array}$ \\
\hline Mirabelie & Hydrogen & $\begin{array}{l}4.5-\mathrm{m} \text { length } \\
1.6 \cdot \mathrm{m} \text { diamcter }\end{array}$ & & $\begin{array}{l}\text { Experiment at } \\
\text { Ecrpulthov in } \\
\text { late } 1971\end{array}$ \\
\hline $\mathrm{BEBC}$ & Hydrogen & $\begin{array}{l}3.7-\mathrm{m} \text { diameter } \\
2.0-\mathrm{m} \text { height }\end{array}$ & .. & Experiment in 1972 \\
\hline BNL 11-foot & Hydrogen & 11-foot sphere & & $\begin{array}{l}\text { Experiment in } \\
\text { summer } 1972\end{array}$ \\
\hline MAI $14=f \circ \Omega t$ & HyArngen & $\begin{array}{l}\text { 12-f९pt } \\
\text { plus two-foot } \\
\text { extension in beam } \\
\text { alrection }\end{array}$ & & $\begin{array}{l}\text { First oool down } \\
\text { in summer } 1972\end{array}$ \\
\hline RHEL & Hydrogen & $\begin{array}{l}\text { 1.5-m diameter } \\
1.0-\mathrm{m} \text { height } \\
70 \mathrm{kG} \text { field }\end{array}$ & . & About 1974 \\
\hline
\end{tabular}

Notes: (I) The RHEL chamber is not a large chamber "in the sense of the other entries, but because of its high field over a substantial volume it does represent a new step and is therefore listed here.

(2) The BNL Il-foot is an expanded version of the present 7 -foot test facility. I have not put in the latter in this list because it is not truly a production facility available to users. 


\section{A. Neutrino Physics}

It is evident that neutrino physics is an area in which there is an enormous potential for new knowledge: our present experimental information on weak interactions comes from the study of a few decays, in which we have little choice of energy or momentum transfer, and very limited data on neutrino interactions in complex nuclei and on nucleons. This area of weak interactions unquestionably supplies one of the major scientific justifications for the construction of higher energy accelerators.

Just to fix ideas it may be useful to review some major areas of interest:

(a) "Elastic Processes"

$$
\begin{aligned}
& \nu+\mathrm{n} \rightarrow \mu^{-}+\mathrm{p} \\
& \bar{v}+\mathrm{p} \rightarrow \mu^{+}+\mathrm{n}
\end{aligned}
$$

(b) Resonance production-form factors and study of selection rules

$$
\begin{aligned}
& \nu+p \rightarrow \Delta^{++}(1236)+\mu^{-} \\
& \nu+n \rightarrow \Delta^{+}+\mu^{-} \\
& \bar{v}+\mathrm{n}>\Delta^{-}+\mu^{+} \\
& \bar{v}+\mathrm{p} \rightarrow \Delta^{0}+\mu^{+}
\end{aligned}
$$

(c) Hyperon production and study of $\Delta S / \Delta Q=1$ rule

$$
\begin{aligned}
& \bar{v}+\mathrm{p} \rightarrow \Lambda^{0}+\mu^{+} \\
& \bar{v}+\mathrm{p} \rightarrow \Sigma^{0}+\mu^{+} \\
& \bar{v}+\mathrm{n} \rightarrow \Sigma^{-}+\mu^{+} \\
& \left.\nu+\mathrm{n} \rightarrow \Sigma^{+}+\mu^{-} \quad \text { (violates } \triangle \mathrm{S} / \Delta Q=1\right)
\end{aligned}
$$

(d) Measurements of total $v \mathbb{N}$ and $\bar{v} N$ cross sections as a function of neutrino energy. It is of interest to determine the validity at higher energies of the previously measured total cross section,

$$
\sigma_{v}-(0.8 \pm 0.2) \mathrm{E}_{v} \times 10^{-38} \mathrm{~cm}^{2} / \text { nucleon }
$$


(e) Studies of $d^{2} \sigma / d q^{2} d v$ in the inelastic continum region, where $q^{2}$ is the lepton squared-momentum-transfer and $v$ is the difference between incident and final total laboratory energies of the leptons.

(f) Searches for higher mass intermediate vector bosons.

(g) Other special studies such as, diagonal couplings -- $v_{e}+e \rightarrow v_{e}+e$ neutral currents at high momentum transfer $-v+p \rightarrow v+p$

The first three of the se groups of experiments are well suited to relatively low energy neutrinos $\left(\mathrm{E}_{v}<5 \mathrm{GeV}\right)$ and will undoubtedly be investigated by the 12-foot ANL and 1I-foot BNL chambers and the CERN 3.7-meter chamber. Exploratory work on $(\mathrm{d}),(\mathrm{e}),(\mathrm{f})$ and $(\mathrm{g})$ at CERN will be carried out by the Gargamelle heavy-liquid chamber; as will be shown further the ability to convert gammas and measure total $v$ energies is essential in (d) and (e) and a $\mathrm{C}_{2} \mathrm{~F}_{5} \mathrm{Cl}$ filling of Gargamelle provides this ability to a useful degree.

With these ideas in mind one can now ask for the uplimal characteristica of a chamber suited to this type of work.

(1) Size--In the 1968 NAL Summer Study Report it was stated that the neutrino program demands the largest chamber compatible with (i) available funds, (ji) technical feasibility, and (iii) a not too excessive cosmic ray background. This argument was used to support the 25-foot chamber proposed for NAL. Unfortunately, limitation of funds has provented the authorization of this proposal. Presently the NAL program includes the construction of a chamber in the shape of a 12-foot-diameter sphere of design siwilar to the proposed BNL modifications of the 7-foot facility. Figure 9 shows a diagram of that design; this picture should only be taken as a zeroth order approximation of what the actual NAL chamber will look like. 
The large size is of course required by rate considerations. To illustrate with a numerical example, a recent study by M. L. Stevenson ${ }^{16}$ of an experiment to do deep inelastic scattering above $15 \mathrm{GeV}$ shows an event rate of one event per 50 machine pulses at $2 \times 10^{13}$ protons per pulse with an assumed total cross section of $0.8 \mathrm{H}_{\nu} \times 10^{-38} \mathrm{~cm}^{2}$. If the actual cross section fails to keep rising at this rate the statistics become even worse. Furthermore, Stevenson's estimates assumed the availability of the whole chamber--gamma ray and muon deitectors are both external. If one uses track sensitive targets with neon outside for photon detection, the effective event rate easily goes down another factor of 3 or. 4. The need for large volume is then obvious. (2) Magnetic Field--Roe and Stevenson ${ }^{17}$ have emphasized the importance of high field for neutrino work, particularly at the high energy.end of the neutrino spectrum. The argument is basically that the neutrino energy for each event must be determined from measurements on secondaries of the interaction to a precision of a few percent or better, because of the high sensitivity of the flux to the energy. In addition, if neon is used outside a track sensitive insert, a high field is crucial to the photon energy measurements. The present NAL proposal envisages a 30 kgauss field. The large BNL, CERN and ANL chambers are designed for 30,35 , and 18 kgauss respectively.

(3) Other Features--Neutrinos do not come momentum analyzed. It is therefore essential if one is to go beyond the few $3 \mathrm{C}$ fit reactions to have detectors of neutrals and detectors of the final lepton. Even if one is studying general features such as cross sections and inelastic form factors rather than the characteristics of special event types the necessity to know total energy and momentum transfer requires detection of the muon and the neutralo. 'lihio detection can be accomplislued hy: 
a) Use of a track-sensitive target with neon outside. For example, in the NAI chamber one could use a 1.6-m diameter, 3-m long target. This leaves about two conversion lengths of neon downstream, but provides a hydrogen volume of only $6 \mathrm{~m}^{3}$ instead of $25 \mathrm{~m}^{3}$ for the whole useful volume. Unfortunately this arrangement gives only one nuclear interaction length and is inadequate for muon detection.

b) Shower detectors and muon detectors external to the chamber. The shower detectors in their simplest form might just measure to a few percent the total energy in photons and in a more complex form would give detailed direction and energy information for all $\pi^{\circ} \mathrm{s}$. The muon detector will have to identify which of the outgoing tracks is the muon. An arrangement of this type suggested. by Stevenson ${ }^{16}$ is shown in Fig. 12.

\section{B. Strong Interaction Physics at High Energies}

There exists some difference of opinion as to the role to be played by large bubble chambers in future studies in strong interactions. The difficulties are the tollowing:

(i) Cross sections of non-diffractive channels susceptible to conventional kinematic fitting--probably only the $4 \mathrm{C}$ events--are rapidly decreasing functions of energy. At high energies such processes might have cross sections only in the microbarn range. At the same time, one would have to measure a very large number of ovente to have a uaeful sample of the fittalie types.

(ii) At the same time there are competing techniques, namely streamer chambers and wire chamber spectrometers which can handle particular configurations of events more effectively insofar as statistics and measuring precision are concerned. The hybrid system with a smaller, rapid-cycling chamber is also a serious competitor for certain experiments. 
Yet there are some positive factors which should also be kept in mind: (iii) As an exploratory tool to provide first detailed looks at high energy processes, the large chamber will probably be of great usefulness. Remembering that the decay lengths of strange particles in the $50-100 \mathrm{GeV}$ range lie in the 3-7 meters region, the large chamber as a survey device will have a significant detection efficiency advantage over an intermediate chamber in the 2-meter domain. Analytical calculations and Monte Carlo studies have demonstrated that 4-constraint events should be readily handlable in a conventional way up to at least 50 or $100 \mathrm{GeV}$. Lach $^{18}$ has suggested the use of upstream wire chambers to provide high precision directional information on beam particles. (iv) The avility to push the usefulness of the large chamber beyond exploration to the detailed study of high energy, multi-particle processes depends, in my view, largely on the success of the track-sensitive-target technique. If the present ideas can be turned into truly operational techniques without, in the target measurements, loss of precision and without. excescivo difficulties in disentangling photons observed in the neon volume and associating them properly with their parent vertex, the large chamber may assume at high energy the same importance that intermediate chambers have had in the one to several GeV regime.

\section{CONCLUSION}

I must apologize to all the scientists and engineers who have made reports to the Conference for my many omissions. As indicated at the beginning, this presentation is not in any sense a summary, but rather a collection of topics of general interest to one who might be interested in being a bubble chamber user during the next few years. As is abundantly clear, there are now a serles of developments which may tremendously increase the power of the 
bubble chamber, namely, hybrid systems, track-sensitive targets, large volume chambers with more or less high magnetic fields. None of these techniques has yet progressed to the point of giving new results and enlightenment previously unavailable. The next few years will tell if the apparent promise of these methods can be translated into a flood of new physics results. Hopefully we shall know at the time of the next Bubble Chamber Conference. 


\section{REFEREINCES}

1. D. Berley et al., to be published; R. O. Bangerter, UCRL-19244 (1969).

2. I. Budagov et al., Phys. Letters 29B, 525 (1969); I. Budagov et al., Phys. Letters 30B, 364 (1969)。

3. J. Ballam et al., Phys. Rev. Letters 24, 960 (1970).

4. R. Armenterọs et al., $\mathrm{K}^{-} \mathrm{p}$ Cross Sections from 440 to $800 \mathrm{MeV} / \mathrm{c}$, submitted to Nucl. Physics B.

5. Angela Barbaro-Galtieri et al。, private communication.

6. G. Chikovani et al., Phys. Letters 25B, 44 (1967); Benz et al., Phys. Letters 28B; 233 (1968); Baum et al., Phys. Letters 31B, 397 (1970).

7. D. G. Coyne, UCRL-19450; G. Goldhaber et al., Phys. Rev. Letters 23, 1351 (1969).

8. H. Barney, A. Rogers and S. J. St. Lorant, Nucl. Instr. and Methods 74 , 345 (1969); A. Rogers, SLAC Work on Rapid Cycling Chambers, Report to this Conference.

9. W. Walker, Wiscunsin Rapid Cycling Chamber, Report to this Conference.

10. R. C. A. Brown, G. Harigel, H. J. Hilke, and P. Jarmann, Ultrasonic B.C. Development at CERN, Report to this Conference.

11. R. E. Terrell and D. B. Cline, Bull. Am. Phys. Soc. 15, 493 (1970); D. Cline and T. Buhl, Bull. Am. Phys. Soc. 15, 513 (1970).

12. B. Gunderson et al., Neutral Particle Tagging Facility for the ANL 30-Inch Bubble Chamber, Keport to this Conference.

13. D. Barisli el al., STAC Froposal No. BC-25.

14. These various systems were discussed by (i) W. Smart, (ii) I. Pless, and (iii) G. Kalbfleisch respectively at this Conference.

15. R. Floreit et al., Nucl. Instr: and Melluds 20, $160 \cdot(1967)$.

16. M. L. Stevenson, Neutrino Physics with Large $\mathrm{H}_{2}$ Chambers, Report to this Conference. 
17. B. Roe and M. L. Stevenson, NAL Summer Study Report sslo6 (1969).

18. J. Lach, Strong Interactions at High Energies, Report to this Conference.

\section{FIGURE CAPTIONS}

Fig. 1. $\mathrm{q}^{2}$ and $\mathrm{M}^{2}\left(\pi^{+} \mathrm{p}\right)$ distributions for events $v:+\mathrm{p} \rightarrow \mu^{-}+\mathrm{p}+\pi^{+}$identi-

fied in the CERN heavy liquid bubble chamber filled with propane.

Fig. 2. $\rho^{\circ}$ decay angular distributions for $r_{p} \rightarrow \rho^{\circ} p$ using linearly polarized photons.

Fig. 3. $\mathrm{K}^{-} \mathrm{p}$ channel cross sections as a function of momentum between 400 and $800 \mathrm{MeV} / \mathrm{e}$.

Fig. 4. (a) $\pi^{+} \cdot \pi^{+} \pi^{-}$mass distribution from ahnut. $5 n$, non events of the type $\pi^{+} \mathrm{p} \rightarrow \mathrm{p} \pi^{+} \pi^{+} \pi^{-}$at $7 \mathrm{GeV} / \mathrm{c}$ incident momentum. (b) Same mass distribution after removal of $\Delta(1236)$ and removal of events with $t_{p p}<0.2(\mathrm{GeV} / \mathrm{c})^{2}$. (c) $\eta \pi^{+}$and $\mathrm{K}^{+} \mathrm{K}^{0}$ mass distribution from the reaction $\pi^{+} \mathrm{p} \rightarrow \pi^{+} \eta p$ and $\pi^{+} \mathrm{p} \rightarrow \mathrm{K}^{+} \overline{\mathrm{K}}^{\mathrm{O}} \mathrm{p}$ respectively.

Fig. 5. $\pi^{+} \pi^{-}$mass spectrum tor the reaction $\pi^{+} p \stackrel{\dot{m}}{\rightarrow} \Delta^{++} \pi^{+} \pi$ near $3.7 \mathrm{GeV} / \mathrm{c}$ incident momentum.

Fig. 6. Setup of Wisconsin group for hybrid system triggering ANL chamber un fast forward neutral secondaries.

Fig. 7. Setup proposed by Cal Tech group for SLAC experiment.

Fig. 8. $r$ mass spectrum in mixture of $2 / 3$ neon, $1 / 3$ hydrogen in BNL 80 -inch chamber.

Fig. 9. Proposed track-sensitive-target arrangement for the 3-7-m European bubble chamber (BEBC).

Fig. 10. Proposed track-sensitive-target configuration for BNL 80-inch chamber. Fig. 11. Rough approximation of proposed NAI 12-foot chamber design.

Fig. 12. Arrangement proposed for studying neutrino deep inelastic scattering at NAL. Quantameter measures total energy in $\pi^{\circ}$ meson. Hadrometer identifies the muon secondary. 


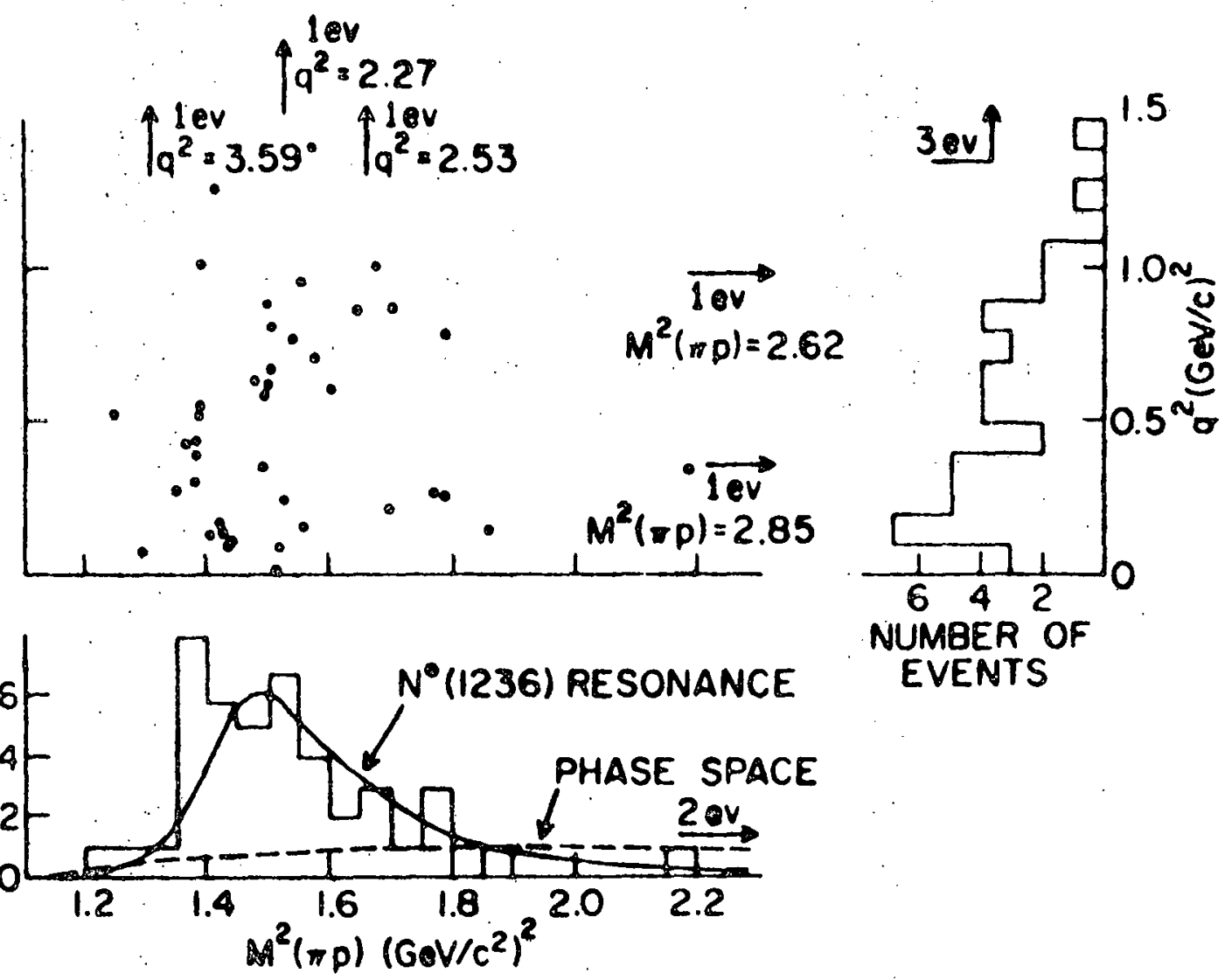

Fig. 1. $q^{2}$ and $M^{2}(\pi p)$ distributions for $\left(\mu^{-} \pi^{+} p\right)$ events with $E_{\text {VIS }}>1 \mathrm{GeV}$. 


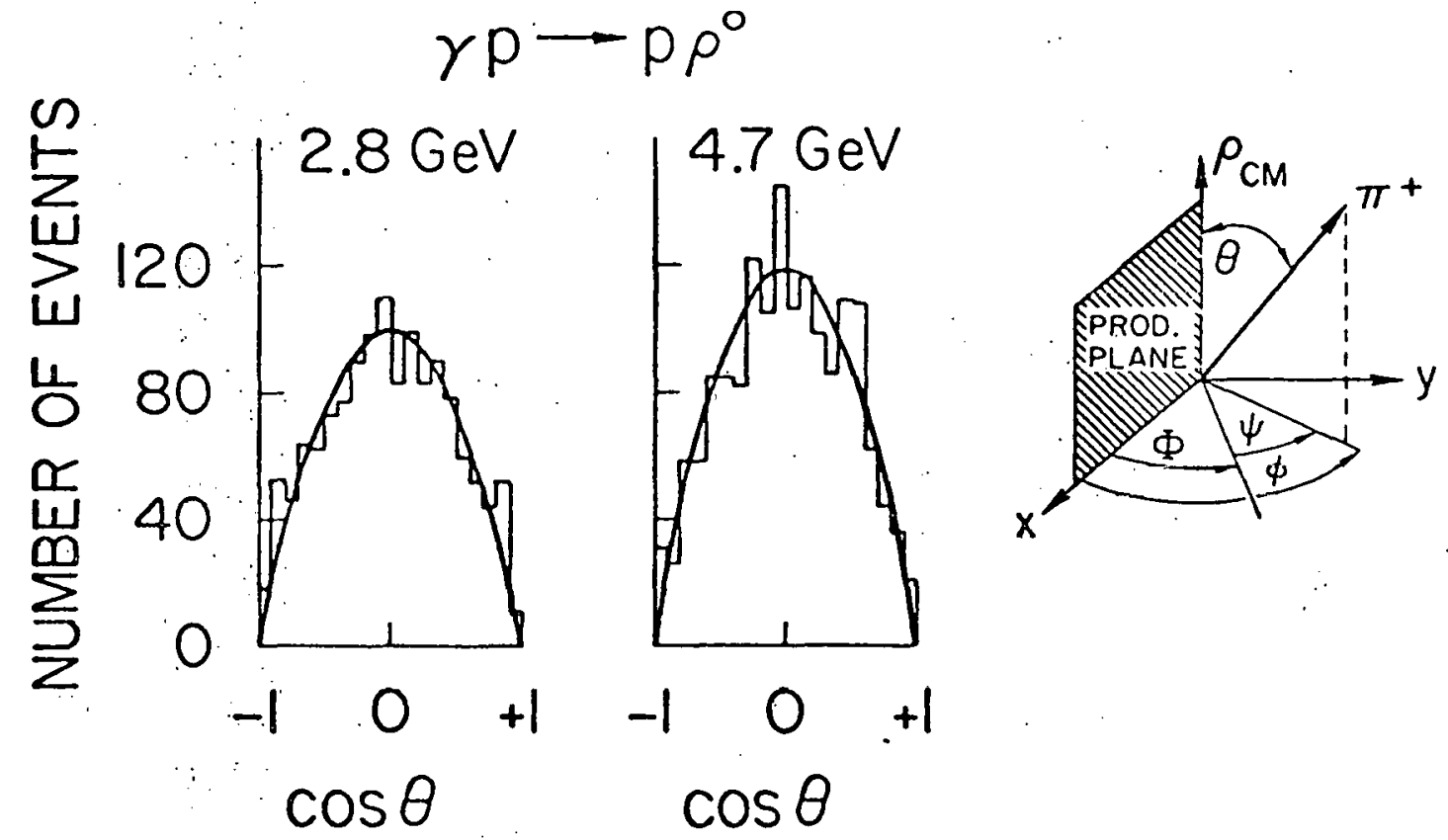

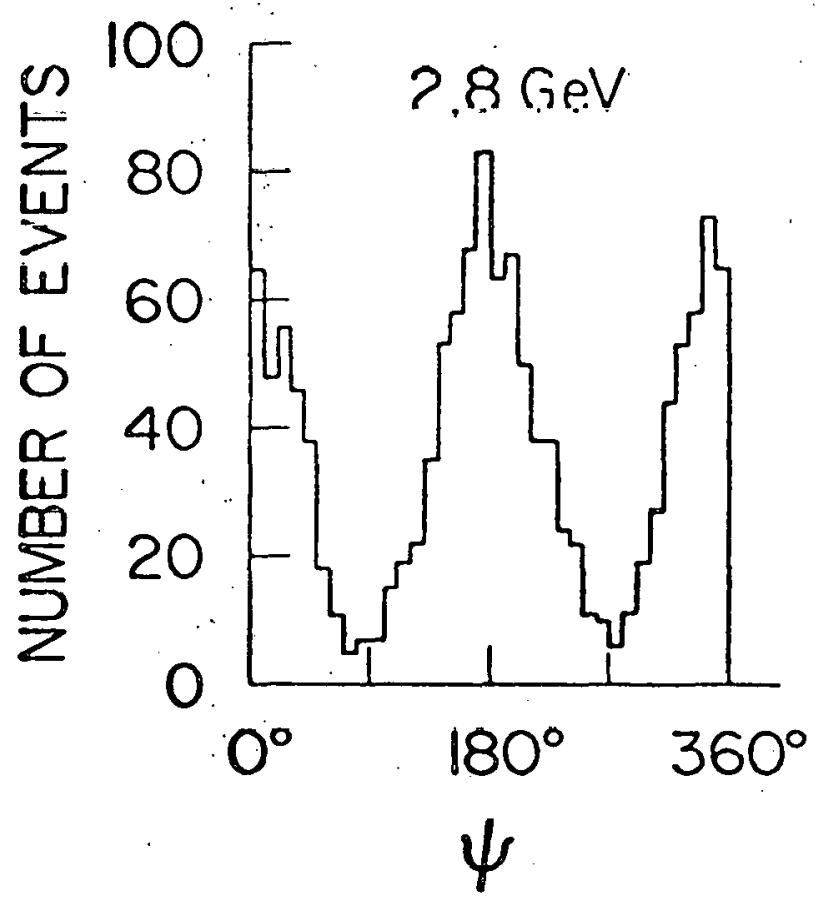

Fig. 2

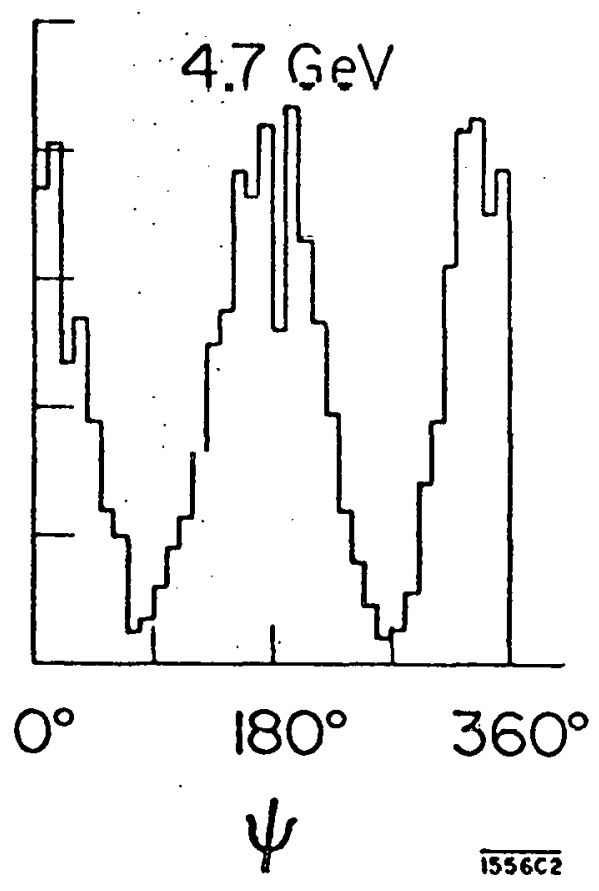

XBL 706-1304 


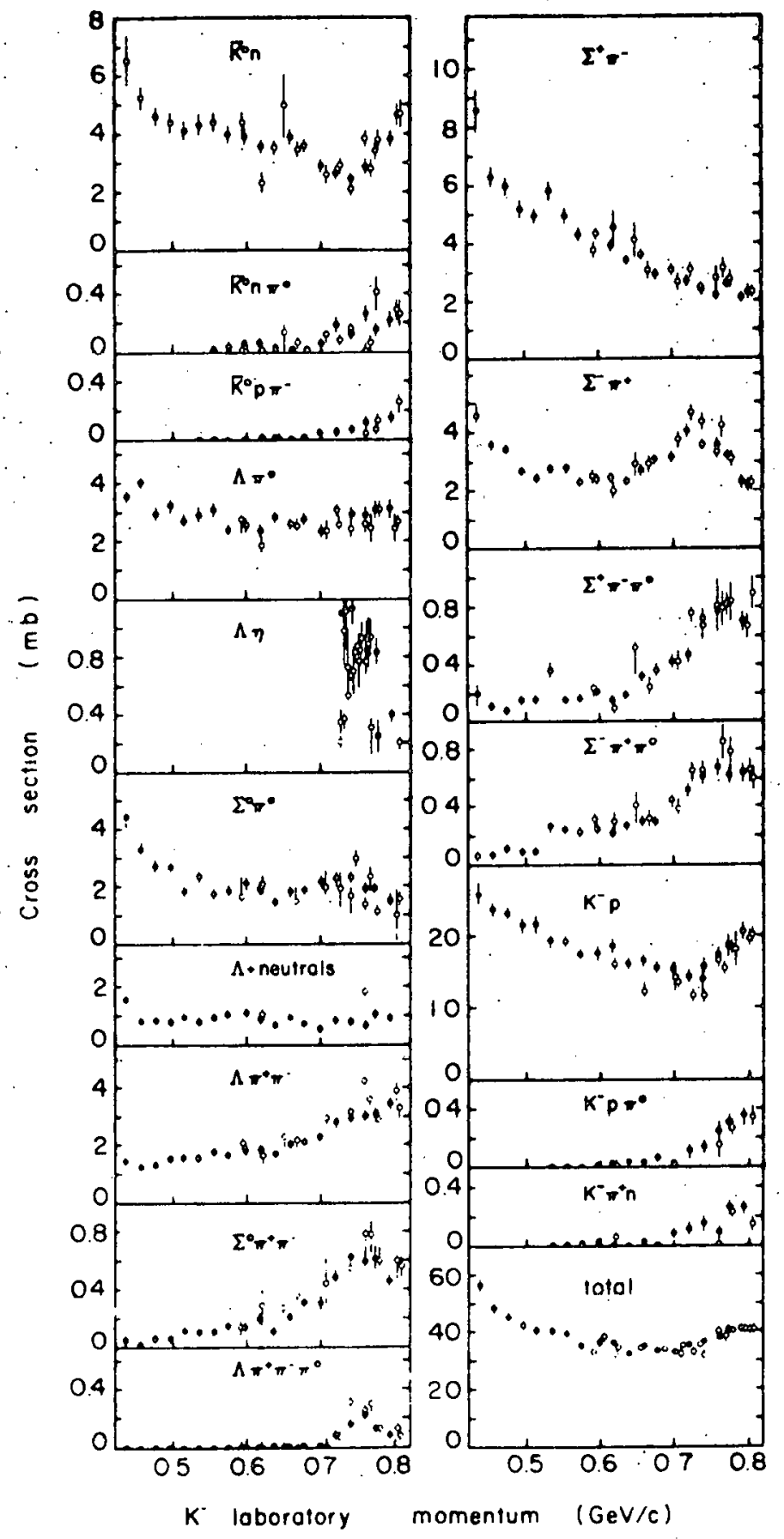

XBL 705-1002

F1E 3 


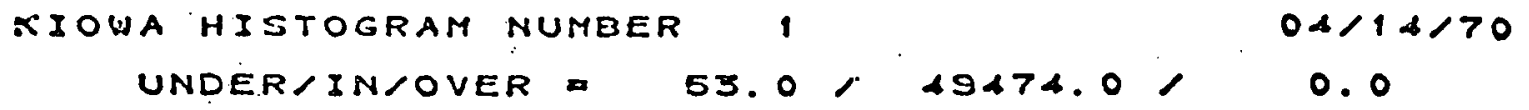
$M(3 P I)$ ALL EVENTS

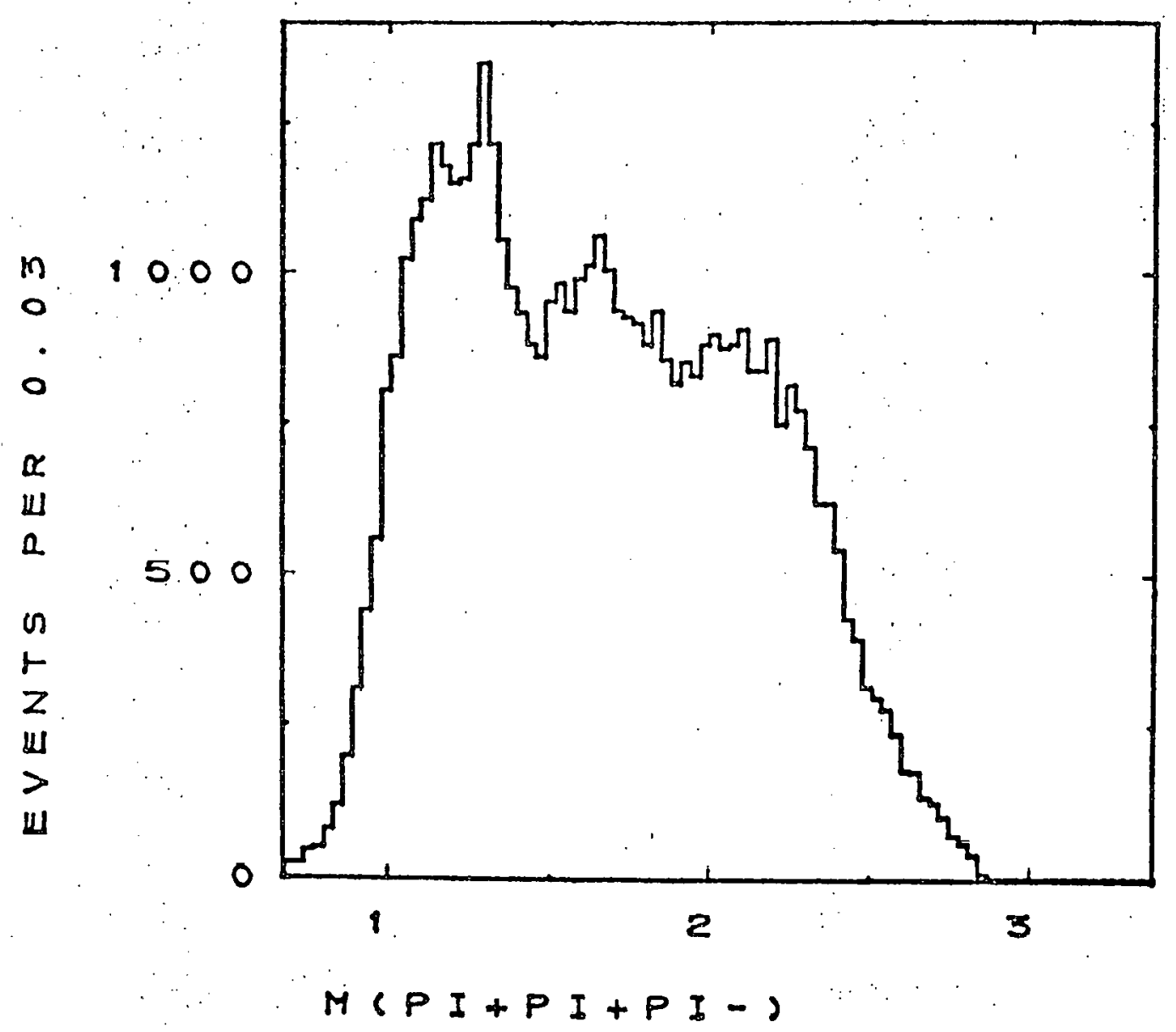

XBL 705-1007

f'ig. $4 a$ 


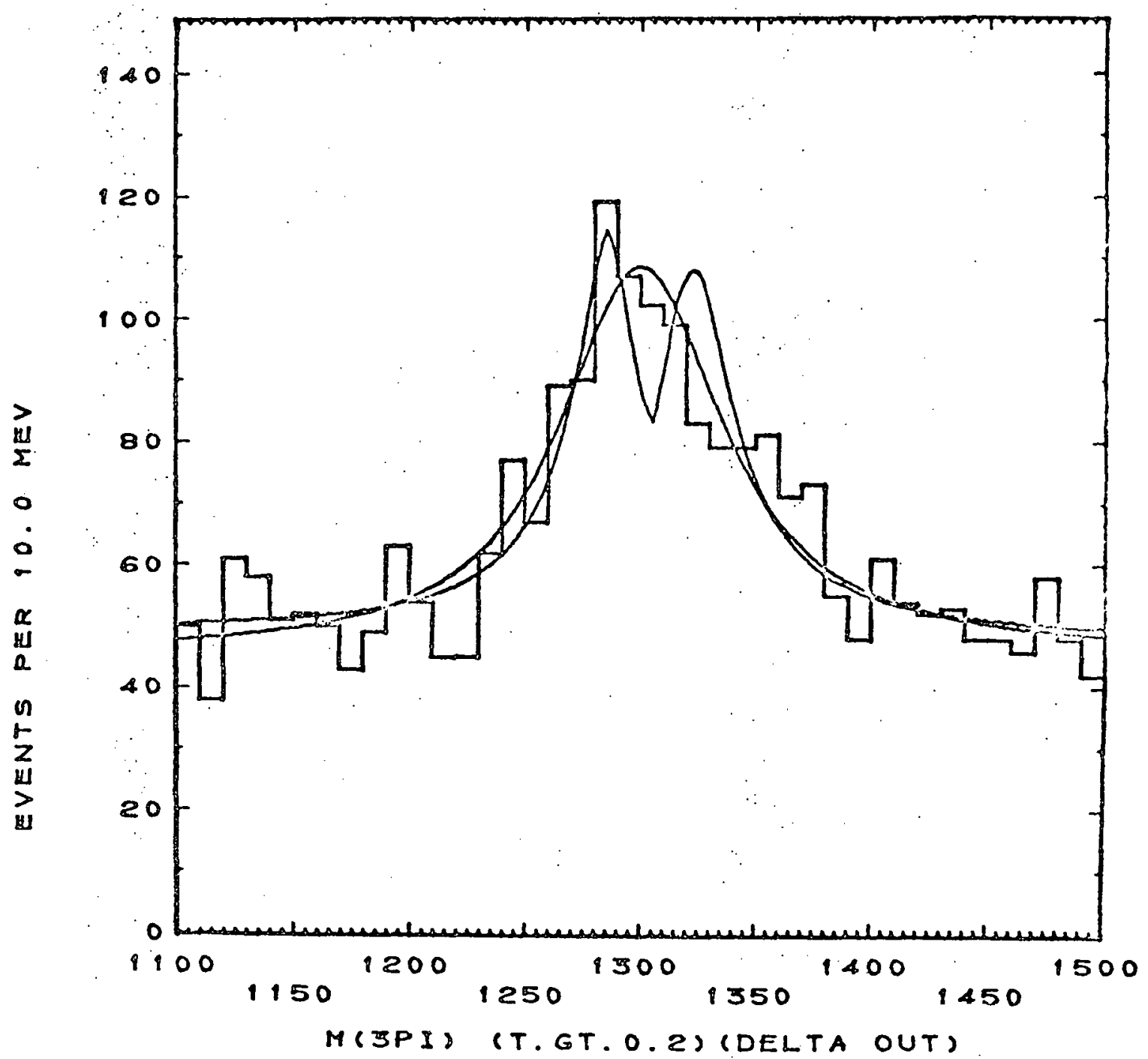

Fig. $4 b$ 
SUM OF PI+ETA ANDK+KO

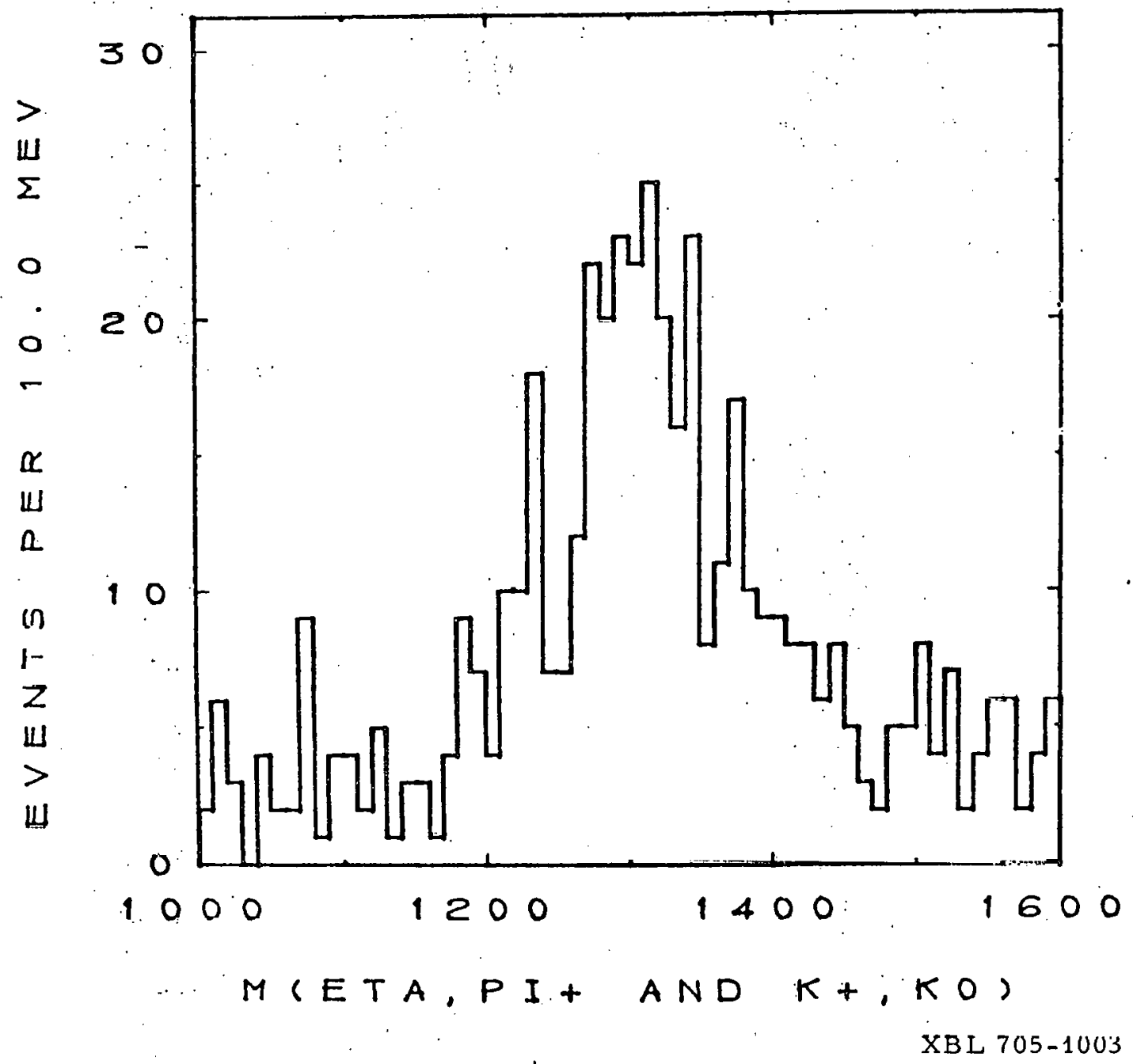

Fig. $4 c$ 

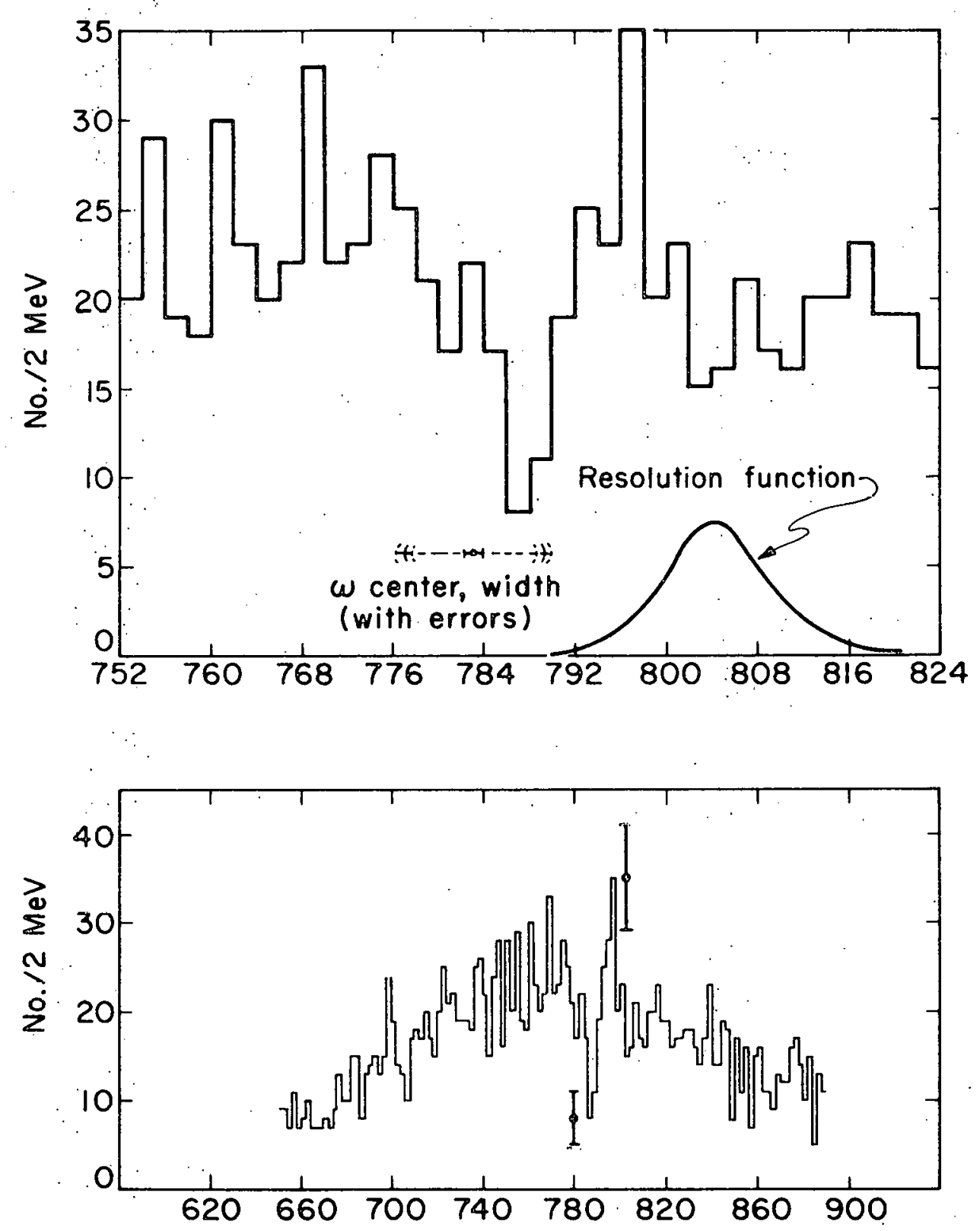

Fig. 5 


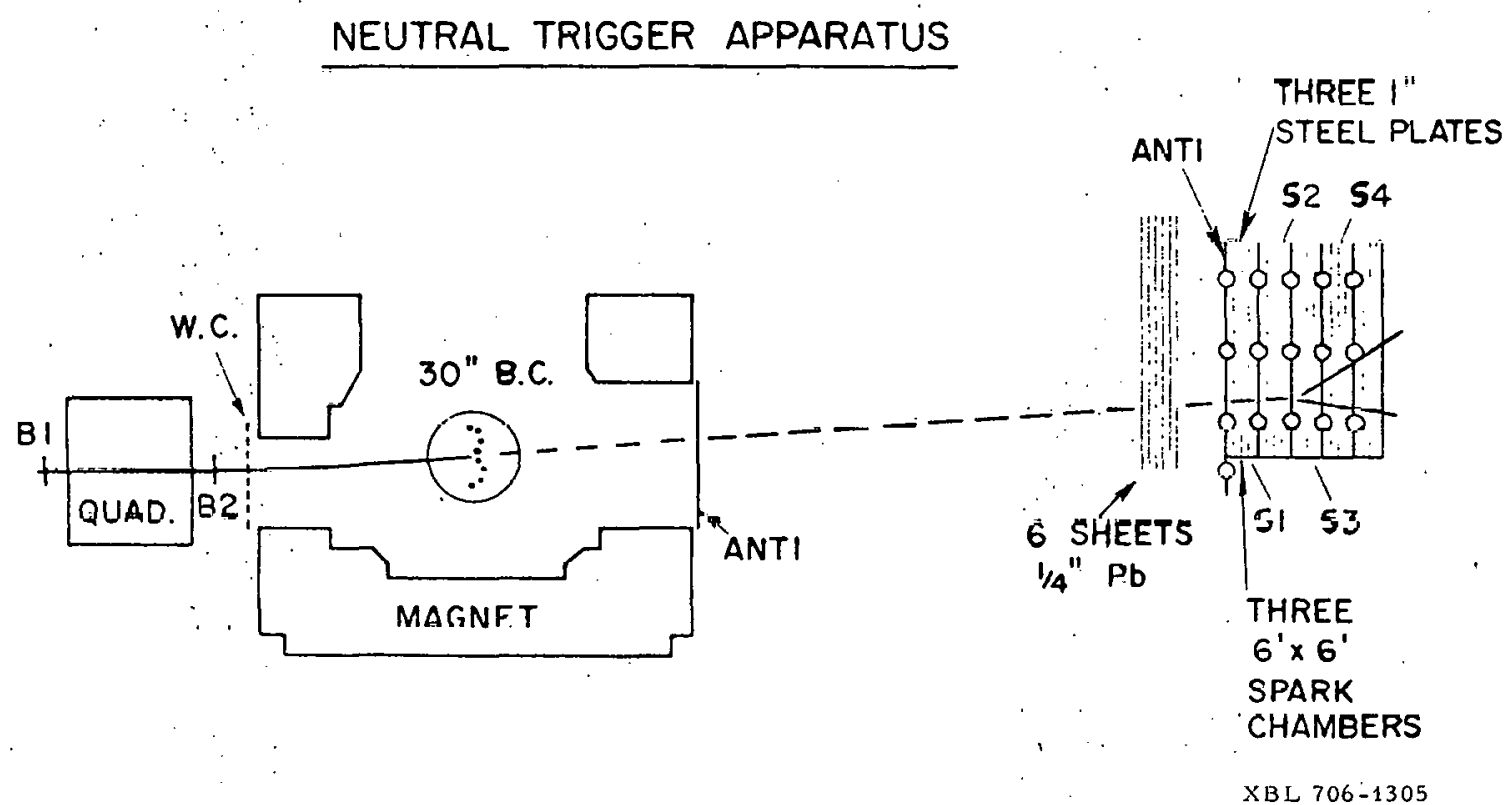

Fig. 6 
Seumgte of apparatus

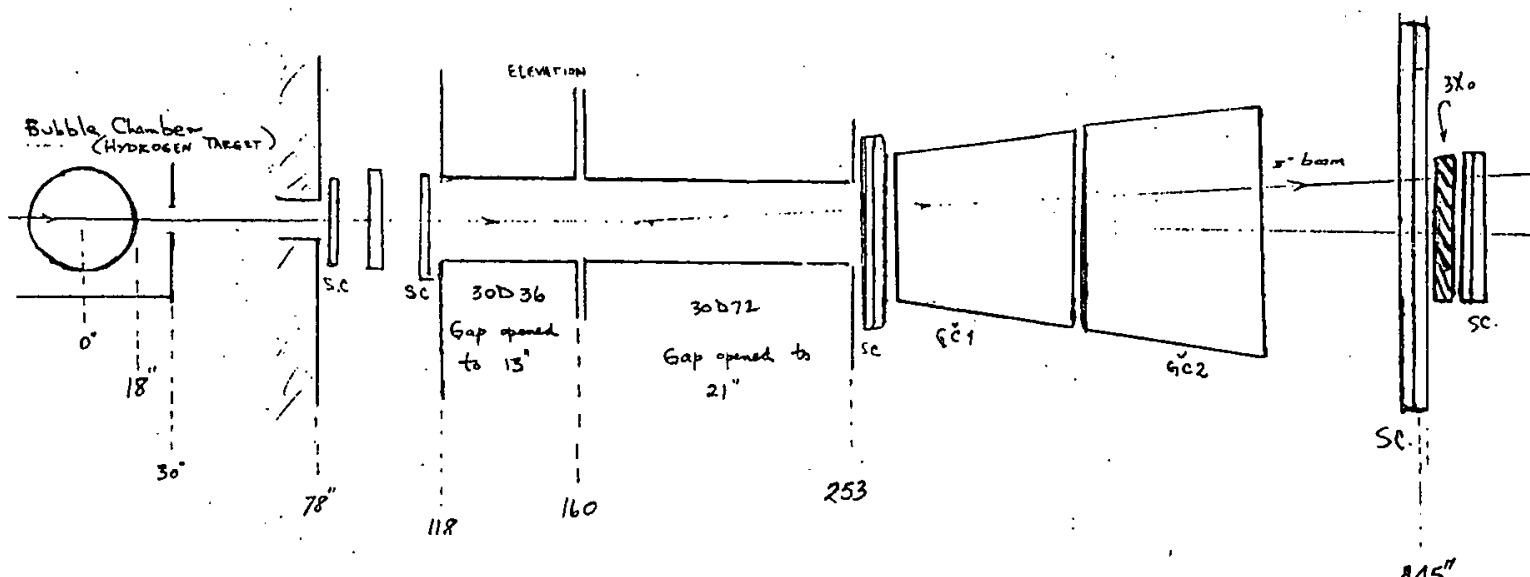

Fig. 7

XBL 706-1043 

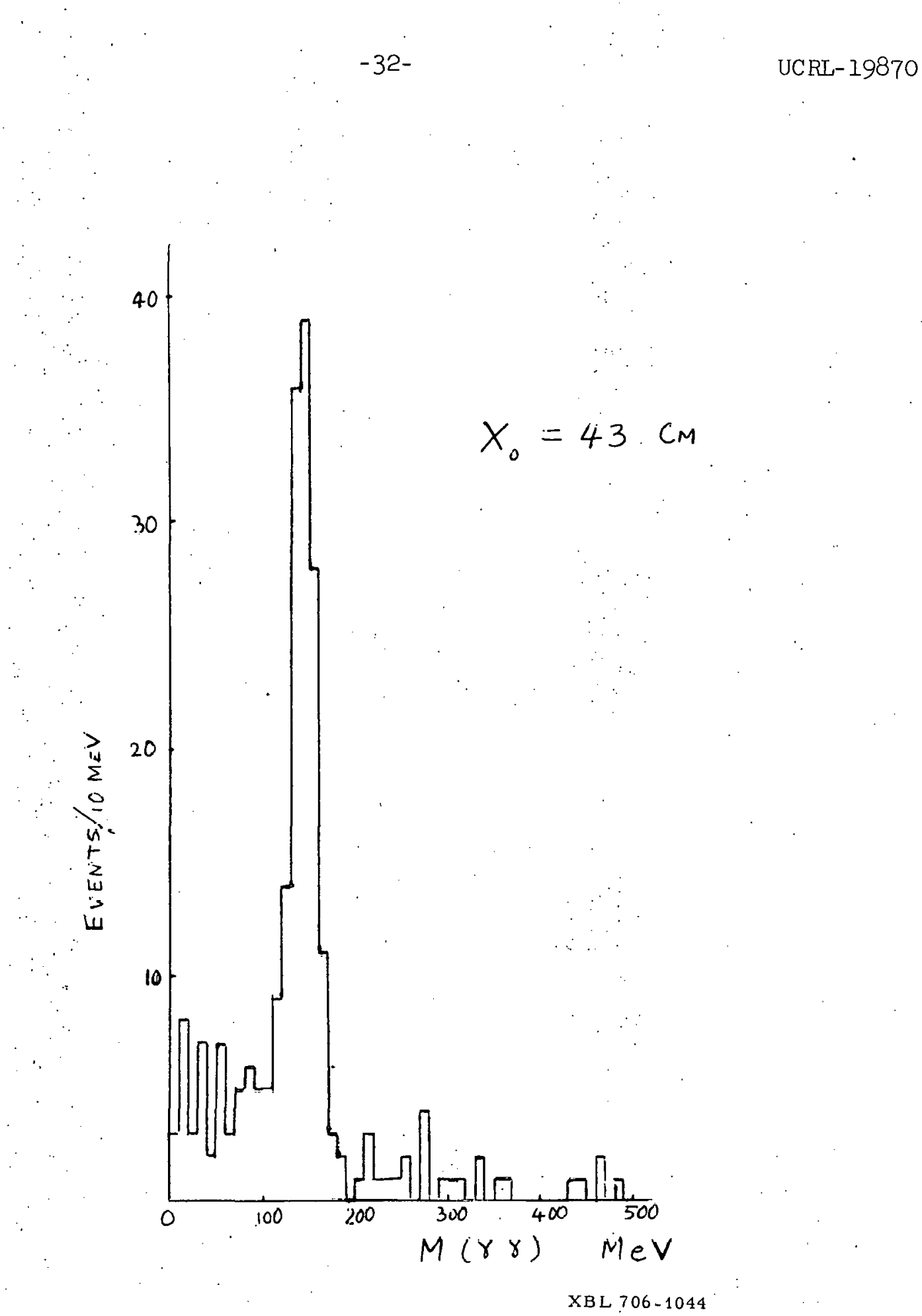

Fig. 8 


$$
(9)
$$




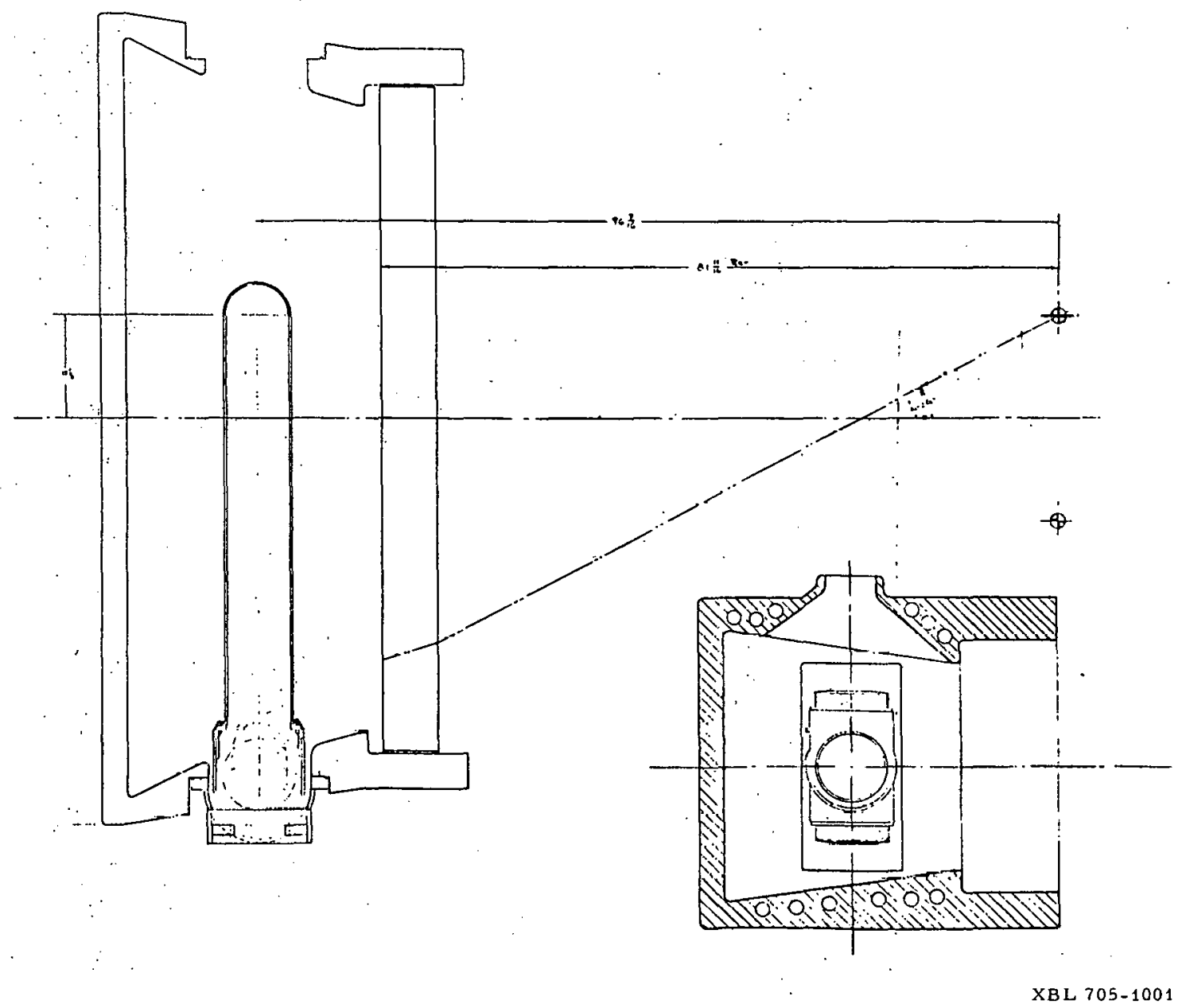

Fig. 10 


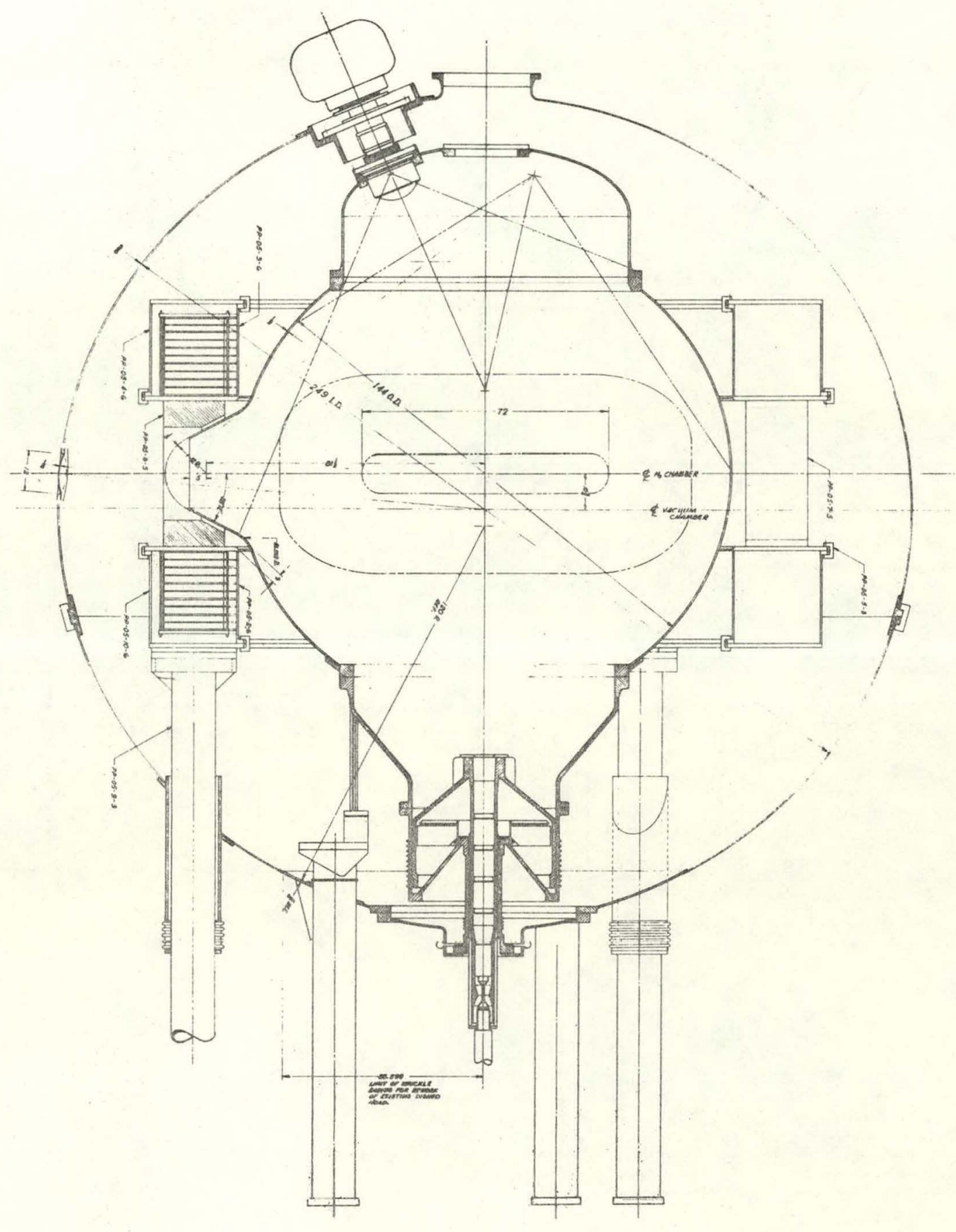

XBL 705-1004

Fig. II 


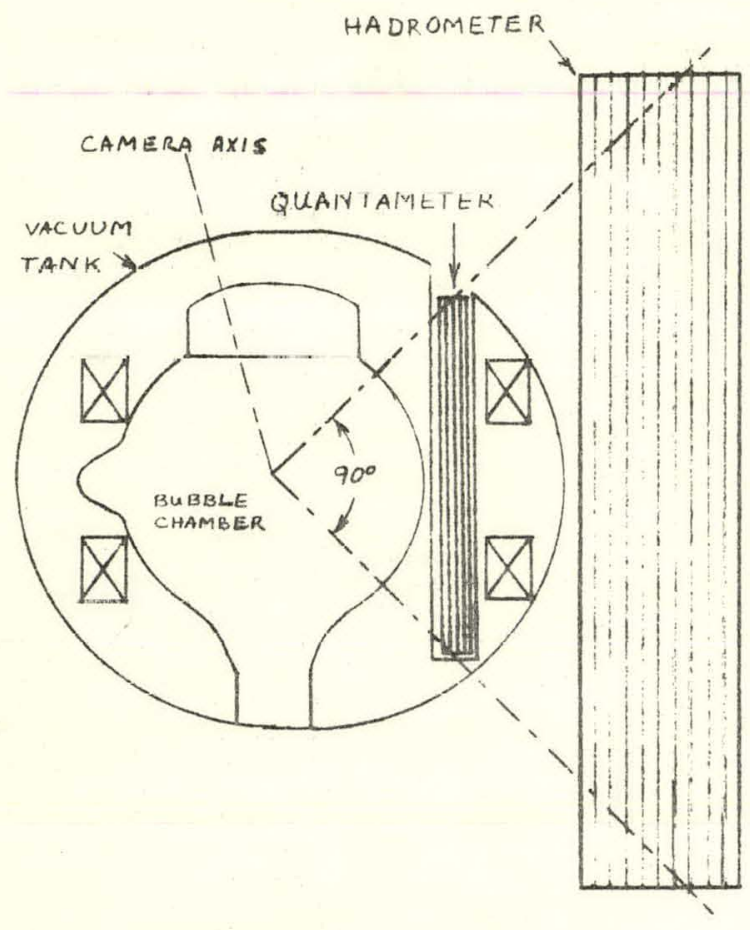

SIDE VIEW

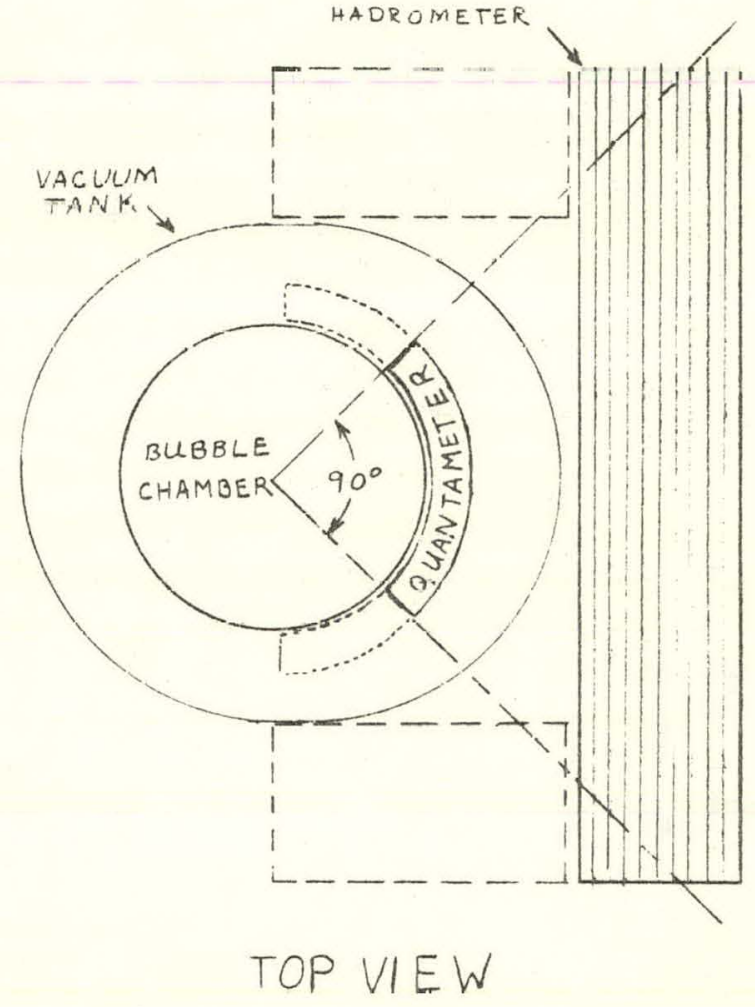

XBL 706-1306

Fig. 12 


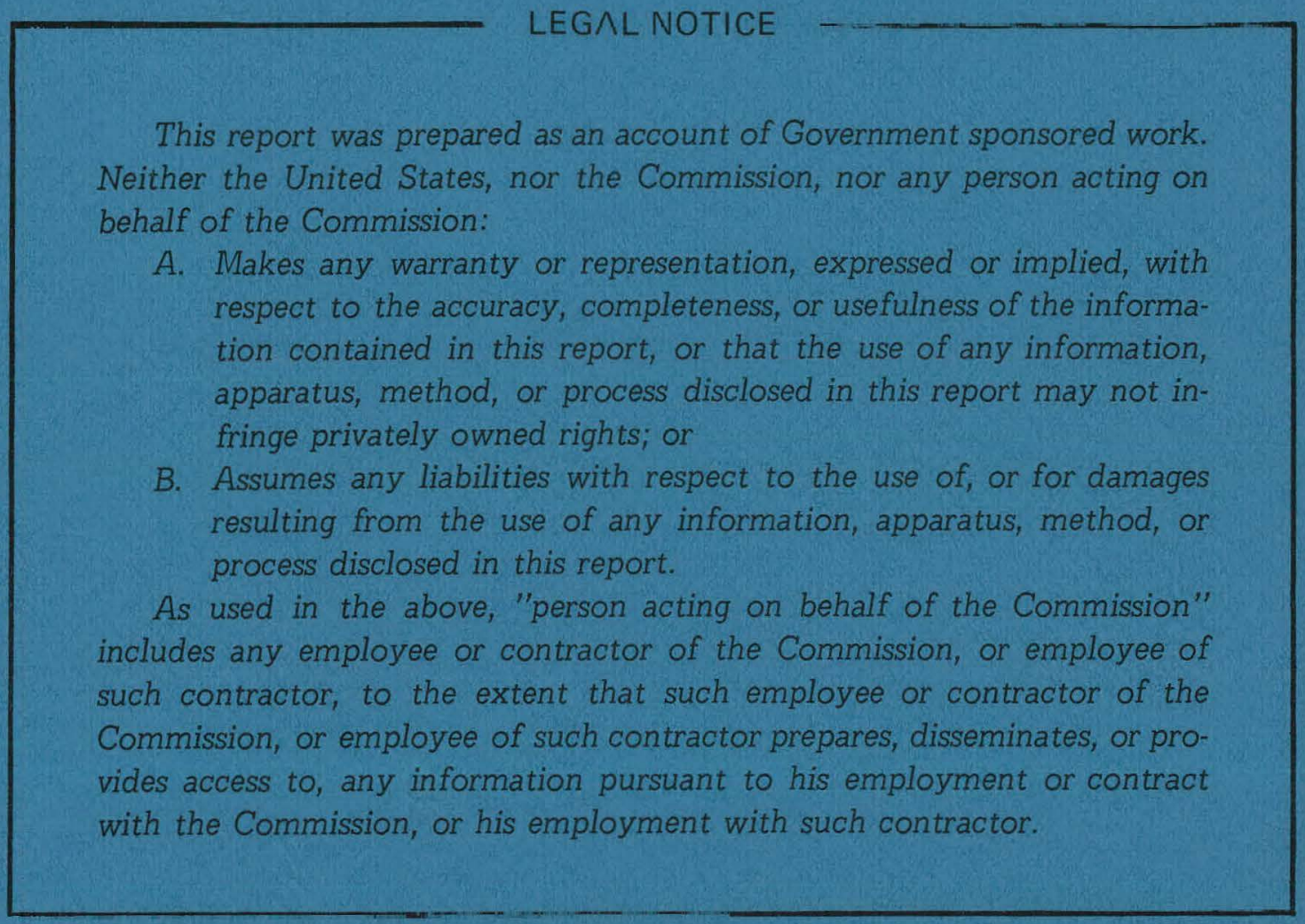


